\title{
Modelling the Type Ic SN 2004aw: a moderately energetic explosion of a massive $\mathrm{C}+\mathrm{O}$ star without a GRB
}

\author{
P. A. Mazzali, ${ }^{1,2 \star}$ D. N. Sauer, ${ }^{3}$ E. Pian,,${ }^{4,5}$ J. Deng, ${ }^{6}$ S. Prentice, ${ }^{1}$ S. Ben Ami, ${ }^{7}$ \\ S. Taubenberger ${ }^{2,8}$ and K. Nomoto ${ }^{9}$ \\ ${ }^{1}$ Astrophysics Research Institute, Liverpool John Moores University, IC2, 134 Brownlow Hill, Liverpool L3 5RF, UK \\ ${ }^{2}$ Max-Planck-Institut für Astrophysik, Karl-Schwarzschild-Str. 1, D-85748 Garching bei München, Germany \\ ${ }^{3}$ German Aerospace Center (DLR), Institute of Atmospheric Physics, D-82234 Oberpfaffenhofen, Germany \\ ${ }^{4}$ IASF-Bo, via Gobetti 101, I-40129 Bologna, Italy \\ ${ }^{5}$ Scuola Normale Superiore, Piazza dei Cavalieri, 7, I-56126 Pisa, Italy \\ ${ }^{6}$ National Astronomical Observatories, CAS, 20A Datun Road, Chaoyang District, Beijing 100012, China \\ ${ }^{7}$ Smithsonian Astrophysical Observatory, 60 Garden St., Cambridge, MA 02138, USA \\ ${ }^{8}$ European Southern Observatory, Karl-Schwarzschild-Str. 2, D-85748 Garching bei München, Germany \\ ${ }^{9}$ IPMU, Kashiwa, 277-8583, Japan
}

Accepted 2017 April 21. Received 2017 April 20; in original form 2017 March 10

\begin{abstract}
An analysis of the Type Ic supernova (SN) 2004aw is performed by means of models of the photospheric and nebular spectra and of the bolometric light curve. SN 2004aw is shown not to be 'broad-lined', contrary to previous claims, but rather a 'fast-lined' SN Ic. The spectral resemblance to the narrow-lined Type Ic SN 1994I, combined with the strong nebular [O I] emission and the broad light curve, points to a moderately energetic explosion of a massive $\mathrm{C}+\mathrm{O}$ star. The ejected ${ }^{56} \mathrm{Ni}$ mass is $\approx 0.20 \mathrm{M}_{\odot}$. The ejecta mass as constrained by the models is $\sim 3-5 \mathrm{M}_{\odot}$, while the kinetic energy is estimated as $E_{\mathrm{K}} \sim 3-6 \times 10^{51} \mathrm{erg}$. The ratio $E_{\mathrm{K}} / \mathrm{M}_{\odot}$, the specific energy that influences the shape of the spectrum, is therefore $\approx 1$. The corresponding zero-age main-sequence mass of the progenitor star may have been $\sim 23-28 \mathrm{M}_{\odot}$. Tests show that a flatter outer density structure may have caused a broad-lined spectrum at epochs before those observed without affecting the later epochs when data are available, implying that our estimate of $E_{\mathrm{K}}$ is a lower limit. SN 2004aw may have been powered by either a collapsar or a magnetar, both of which have been proposed for gamma-ray burst SNe. Evidence for this is seen in the innermost layers, which appear to be highly aspherical as suggested by the nebular line profiles. However, any engine was not extremely powerful, as the outer ejecta are more consistent with a spherical explosion and no gamma-ray burst was detected in coincidence with SN 2004aw.
\end{abstract}

Key words: line: formation - line: identification - radiative transfer-gamma-ray burst: general-supernovae: general - supernovae: individual: SN 2004aw.

\section{INTRODUCTION}

Type Ic supernovae ( $\mathrm{SNe}$ ) are $\mathrm{H}$-/He-poor core-collapse $\mathrm{SNe}$ (Clocchiatti \& Wheeler 1997; Filippenko 1997; Matheson et al. 2001; Modjaz et al. 2016). Significant diversity can be found among the He-poor $\mathrm{SNe}$ whose data have been published (Bianco et al. 2014; Taddia et al. 2015; Lyman et al. 2016; Prentice et al. 2016). For example, models of the gamma-ray burst (GRB) SN 1998bw (Iwamoto et al. 1998) indicate that it ejected $M_{\mathrm{ej}} \sim 10 \mathrm{M}_{\odot}$ of material with kinetic energy $E_{\mathrm{K}} \sim 4 \times 10^{52} \mathrm{erg}$, and synthesized

${ }^{\star}$ E-mail: P.Mazzali@1jmu.ac.uk $\sim 0.4 \mathrm{M}_{\odot}$ of ${ }^{56} \mathrm{Ni}$, which powered the light curve. These results suggested a progenitor of $\sim 40 \mathrm{M}_{\odot}$. In contrast, SN 1994I (Richmond et al. 1996) was less luminous and energetic. Radiation transport models (Sauer et al. 2006) show that it ejected only $\sim 1 \mathrm{M}_{\odot}$ of material with $E_{\mathrm{K}} \sim 10^{51} \mathrm{erg}$. Both the $E_{\mathrm{K}}$ and the mass of ${ }^{56} \mathrm{Ni}$ synthesized by SN 1994I $\left(M_{\mathrm{Ni}} \sim 0.08 \mathrm{M}_{\odot}\right)$ are similar to ordinary SNe IIP, which suggests a progenitor mass of $\sim 15 \mathrm{M}_{\odot}$. A star of this mass must undergo severe envelope stripping to result in anything other than a SN IIP. Hachinger et al. (2012) showed that small amounts of $\mathrm{He}$ and $\mathrm{H}$ are sufficient to transform a spectrum from $\mathrm{H}$-/He-poor to $\mathrm{H}$-poor/He-rich and from $\mathrm{H}$-poor/He-rich to $\mathrm{H}-/ \mathrm{He}-\mathrm{rich}$, respectively. How this mass-loss occurs is not fully understood, but strong binary interaction is considered to be the 
most likely way to strip a progenitor of its outer layers (Nomoto, Iwamoto \& Suzuki 1995; Eldridge et al. 2013).

Given the large range of $M_{\mathrm{ej}}$ of SNe Ic, we can infer that a wide range of progenitor stars can end their lives as SNe Ic, depending on their evolution. Many of the best observed SNe Ic are luminous, massive and energetic, and belong to an extreme subclass sometimes called hypernovae. These are characterized by early spectra showing very broad absorption features indicative of very high ejecta velocities (Mazzali et al. 2002, 2010). Some of these SNe have been discovered in coincidence with long-duration GRBs (e.g. Galama et al. 1998; Mazzali et al. 2006a; Ashall et al. 2017). While the collapsar scenario, involving the formation of a black hole and the subsequent accretion on to it of additional material from the stellar core (MacFadyen \& Woosley 1999; Woosley \& Bloom 2006; Fryer et al. 2007), has enjoyed much success, the fact that all GRB-SNe have $E_{\mathrm{K}} \sim 10^{52} \mathrm{erg}$, and that the collimation-corrected energy of the associated GRBs is always much smaller than the $\mathrm{SN} E_{\mathrm{K}}$, has been suggested to indicate that GRB-SNe could be energized by magnetars (Mazzali et al. 2014; Greiner et al. 2015; Ashall et al. 2017). SN 2006aj, which was associated with an X-ray flash, was modelled as the explosion of a star of $M_{\text {ZAMS }} \sim 20 \mathrm{M}_{\odot}$, and a magnetar was claimed to have energized the explosion also in this case (Mazzali et al. 2006b). Magnetars may therefore be responsible for most, perhaps all $\mathrm{SNe}$ associated with relativistic events, which would be in keeping with the original suggestion that the collapsar is a failed SN (MacFadyen \& Woosley 1999). We also know SNe that have a high-velocity, high-energy tail to their ejecta, which causes broad absorption lines, but were not associated with a GRB (e.g. SN 1997ef; Mazzali, Iwamoto \& Nomoto 2000). In some cases, the mass and energies involved may have been too small (e.g. SN 2002ap; Mazzali et al. 2002); in other cases, the orientation of the event may have been unfavourable for the detection of a possible GRB (e.g. SN 2003jd; Mazzali et al. 2005; Valenti et al. 2008).

Extracting SN properties requires good observational data as well as explicit modelling of these data (spectra and light curves), which implies some amount of work and has been done for only a very small number of SNe. Although significant uncertainties affect the estimates of mass and energy obtained even with this method, Mazzali et al. (2013) show that properties derived using scaling arguments can easily lead to much larger uncertainty, while properties derived using simplistic approaches are not to be trusted at all. It is therefore important not only to extract the properties of the most extreme $\mathrm{SNe}$, but to map the entire range of $M_{\mathrm{ej}}, E_{\mathrm{K}}$ and ${ }^{56} \mathrm{Ni}$ mass in order to understand how SNe Ic are produced and what is the relation to other types of stripped-envelope $\mathrm{SNe}$, which may show different distributions of properties (e.g. Prentice \& Mazzali 2017).

SN 2004aw is an interesting SN to study because it appears to be an intermediate case and it was very well observed (Taubenberger et al. 2006). Under the classification scheme presented in Prentice \& Mazzali (2017), it is classified as an SN Ic6. It was spectroscopically similar to SN 1994I and PTF12gzk (Ben-Ami et al. 2012), which was characterized by high line velocities $\left(>20000 \mathrm{~km} \mathrm{~s}^{-1}\right)$. SN 2004aw is intermediate between these two $\mathrm{SNe}$ in velocity. In Fig. 1, we compare near-maximum spectra of the three SNe. Despite the large velocity difference between the spectra, it is apparent that none of them show the extreme line blending that is typical of broad-lined SNe Ic. The light curve of SN 2004aw is somewhat broader than that of SN 1994I, and depending on the reddening that is assumed, it may also be more luminous. SN 2004aw may therefore represent an intermediate type of event, and

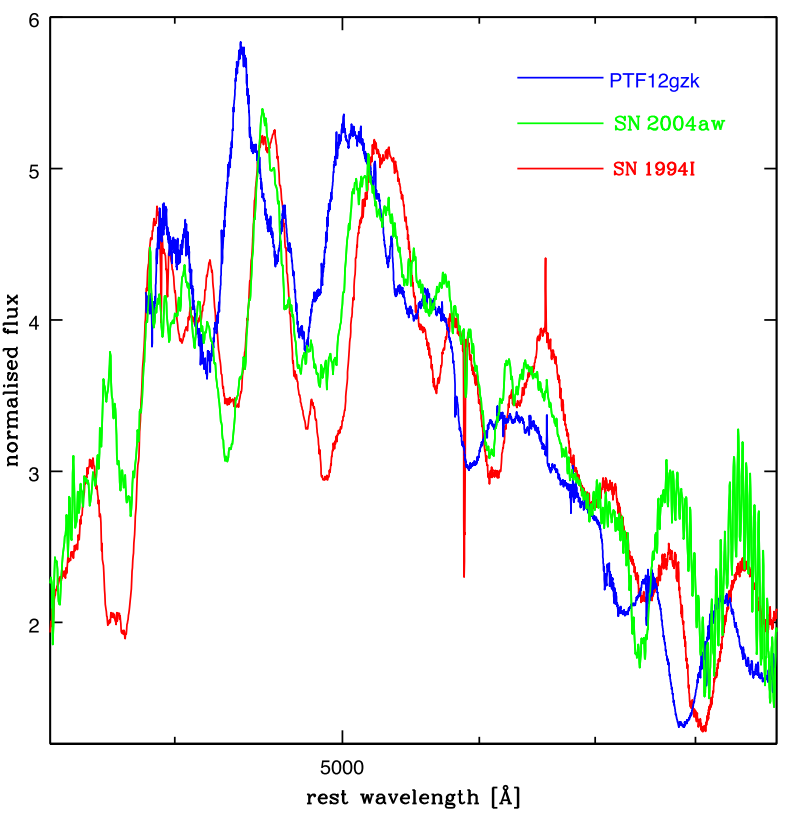

Figure 1. Spectra of SN 2004aw (2004 March 24, 1 d after $B$-band maximum), 1994I (1994 April 4, 4 d before $B$-band maximum) and PTF12gzk (2012 August 2, 2 d before $B$-band maximum), showing the close similarity in spectral shape and line width and the shift in line velocity. PTF12gzk has the highest velocities, and SN 1994I has the lowest, especially if the early epoch of the spectrum shown here is taken into account.

as such it provides a good opportunity to further our understanding of He-poor SNe.

In this paper, we describe our one-dimensional (1D) models for the photospheric and nebular spectra of SN 2004aw, and for the bolometric light curve. Using our 1D radiative transfer codes, we constrain the ejected mass, kinetic energy and ejected ${ }^{56} \mathrm{Ni}$ mass of SN 2004aw, which are then compared with those of other Type Ic $\mathrm{SNe}$.

We analysed SN 2004aw in the spirit of abundance tomography (Mazzali et al. 2014). In Section 2, we describe our models for the early-time spectra, and discuss how they were used to establish a density distribution. We then proceed to the nebular spectra, and show how these can be used to determine the mass and density of the ejecta at low velocities, as well as their composition, in particular with respect to the amount and distribution of ${ }^{56} \mathrm{Ni}$. We also show how the profile of the emission lines can be used to optimize the model (Section 3). We then use the model of the ejecta obtained through spectral modelling, including the distribution of ${ }^{56} \mathrm{Ni}$, to compute a synthetic bolometric light curve, which we compare to the bolometric light curve of SN 2004aw constructed from the available photometry (Section 4). Finally, in Section 5, we discuss our results, and place SN 2004aw in the context of Type Ic SNe.

Following Taubenberger et al. (2006), a distance modulus of $\mu=34.17$ for the host galaxy, NGC 3997, was used. A combined Galactic plus local reddening of $E(B-V)=0.37$ is assumed throughout this paper.

\section{MODELS FOR THE PHOTOSPHERIC SPECTRA}

We modelled a series of photospheric spectra of SN 2004aw using our Monte Carlo SN spectrum synthesis code. Spectra with epoch between 1 and $28 \mathrm{~d}$ after $B$ maximum were selected from Taubenberger et al. (2006). 
Our method is based on a Monte Carlo solution of the line transfer that was developed by Abbott \& Lucy (1985), Mazzali \& Lucy (1993), Lucy (1999) and Mazzali (2000). The models assume an inner boundary ('photosphere') of velocity $v_{\mathrm{ph}}(t)$ for an epoch $t$ relative to the explosion where all radiation is emitted as a blackbody continuum of temperature $T_{\mathrm{BB}}$. This is generally a good assumption for early epochs, and typically works until epochs of 3-4 weeks after maximum for oxygen-dominated SN Ic ejecta. The ejecta are described by a density distribution (an explosion model) and by a composition, which can vary with depth. The gas in the ejecta and the radiation field is assumed to be in radiative equilibrium. Photon energy packets are followed through the ejecta, where they undergo scattering events. For line scattering, a branching scheme based on transition probabilities in the Sobolev approximation allows photons to be emitted in transitions different from those in which they were absorbed. In order to account for the energy of photons that are scattered back into the photosphere (line blanketing), $T_{\mathrm{BB}}$ is adjusted in an iterative procedure to obtain the desired emergent luminosity, $L$. The emergent spectrum is obtained from a formal solution of the transfer equation using the source functions that are derived from the Monte Carlo simulation. The code has been used for a number of SNe Ic (e.g. Mazzali et al. 2013).

Early-time spectra probe the outer part of the ejecta, and can be used to establish both the abundance and the density distribution. In the case of SN 2004aw, the density structure above $5000 \mathrm{~km} \mathrm{~s}^{-1}$ was modelled based on the 1D hydrodynamical model CO21, which was developed for SN 1994I (Iwamoto et al. 1994). The density was scaled up by a factor of 3 in order to achieve enough optical depth in the lines. This approach is justified by the overall spectral similarity between SN 2004aw and SN 1994I at similar photospheric epochs and by the good match of our model spectra to the observed ones (see Fig. 2). This simplistic approach is necessary because of the lack of a grid of explosion models at different masses and energies. On the other hand, rescaling is justified because $\mathrm{CO}$ cores of massive stars tend to have self-similar density profiles even if they differ in mass (Meynet, private communication). This rescaling can lead to some additional uncertainty, so we are very generous with our error estimates below. Homologous expansion is assumed at all times, so the ejecta can be conveniently described using velocity as a comoving coordinate.

As the SN ejecta expand, the line-forming region of the $\mathrm{SN}$ recedes to progressively lower velocities. Subsequent epochs are therefore modelled by adding shells below the previous inner boundary while retaining the density and composition of the layers described by models of earlier epochs, following the approach called 'abundance tomography' (Stehle et al. 2005).

Our models require a time from explosion so that the density structure can be appropriately rescaled. SN 2004aw was discovered late, only $5 \mathrm{~d}$ before $B$ maximum, so a direct determination of the epoch of explosion is not possible. Taubenberger et al. (2006) showed that the light curves of SN 2004aw are broader than those of SN 2002ap, whose rise time was determined through modelling to be $\sim 10 \mathrm{~d}$ (Mazzali et al. 2002). SN 2004aw reached $V$ maximum $\sim 3$ d after $B$ maximum (Taubenberger et al. 2006). Bolometric maximum was intermediate between these two (see Section 4). We adopted for SN 2004aw a rise time to $B$ maximum of $14 \mathrm{~d}$. Epochs used in the models are shown in Fig. 2 and listed in Table 1 along with other modelling parameters. We did not correct for time dilation caused by the small redshift $\left(c z=4906 \mathrm{~km} \mathrm{~s}^{-1}\right)$ since the uncertainty in the epoch (at least $\pm 1 \mathrm{~d}$ ) is larger than the correction.

Fig. 2 shows our best-fitting model spectra (black lines) compared to the observed spectra (grey lines). This ejecta model has $\sim 3 \mathrm{M}_{\odot}$

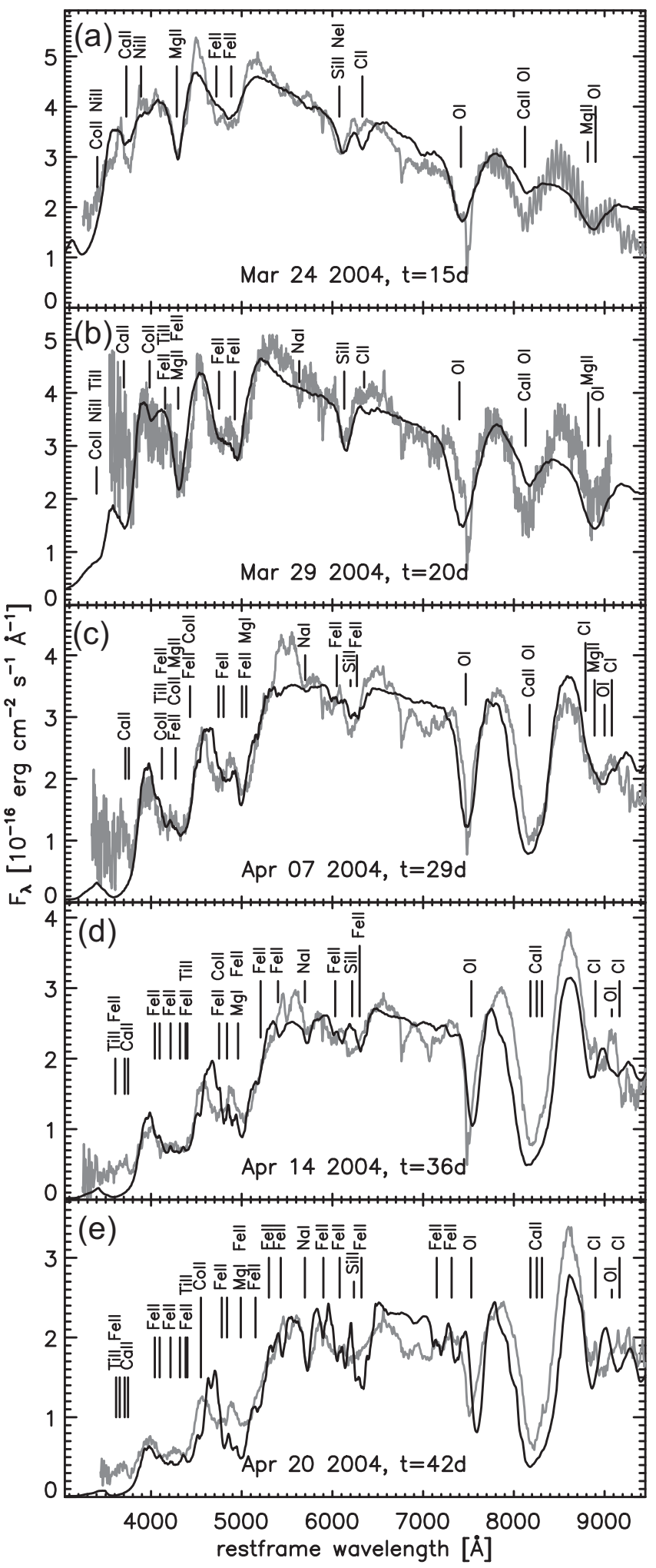

Figure 2. Model spectra (black lines) compared to the respective observed spectra of SN 2004aw (Taubenberger et al. 2006) at different epochs (grey lines). The line labels refer to the most prominent ions causing the respective absorption features. The epochs refer to the time after the assumed explosion date. 
Table 1. Model parameters for the early-time models.

\begin{tabular}{lcccc}
\hline Date & $\begin{array}{c}L \\
(\mathrm{~d})\end{array}$ & $\begin{array}{c}v_{\mathrm{ph}} \\
\left(\mathrm{erg} \mathrm{s}^{-1}\right)\end{array}$ & $\begin{array}{c}T_{\mathrm{BB}} \\
(\mathrm{K})\end{array}$ \\
\hline 2004 March 24 & 15 & $4.04 \times 10^{42}$ & 11100 & $8.9 \times 10^{3}$ \\
2004 March 29 & 20 & $3.65 \times 10^{42}$ & 9600 & $8.0 \times 10^{3}$ \\
2004 April 07 & 29 & $2.97 \times 10^{42}$ & 8100 & $6.6 \times 10^{3}$ \\
2004 April 14 & 36 & $2.30 \times 10^{42}$ & 6600 & $5.9 \times 10^{3}$ \\
2004 April 21 & 43 & $1.91 \times 10^{42}$ & 5000 & $6.0 \times 10^{3}$ \\
\hline
\end{tabular}

of material above $5000 \mathrm{~km} \mathrm{~s}^{-1}$. We also tested ejecta models with different mass in an effort to break the parameter degeneracy that affects the light curve modelling. We found that the SN 2004aw spectra can be reproduced reasonably well only if the density scaling factor of $\mathrm{CO} 21$ is between $\sim 2$ and $\sim 4$. Models with larger mass have excessively high line velocities that cause the absorption features to appear too broad. In particular, the oxygen line at $\sim 7400 \AA$ becomes significantly too broad if too much material is present at high velocities. This line is highly saturated and therefore fairly insensitive to the $\mathrm{O}$ abundance within reasonable limits, meaning that the strength and shape of the absorption feature is primarily set by the density structure. The lower limit for the mass above any photosphere is constrained by the amount of material needed to generate notable P-Cygni features.

In the following paragraphs, we discuss the properties of the individual models in more detail. The primary input parameters for all early-time spectral models are summarized in Table 1.

The first spectrum that we modelled is from 2004 March 24, $1 \mathrm{~d}$ after $B$-band maximum and $t=15 \mathrm{~d}$ after explosion. Fig. 2(a) shows a comparison of the model spectrum to the observed one. The model with $L=4.04 \times 10^{42} \mathrm{erg} \mathrm{s}^{-1}$ and $v_{\mathrm{ph}}=11100 \mathrm{~km} \mathrm{~s}^{-1}$ reproduces most of the observed features, although the re-emission features are not strong enough in some places. All Ca II absorptions, the H\&K feature at $\sim 3750 \AA$ and the IR triplet at $\sim 8100 \AA$, are somewhat weaker in the model than in the observation. To fit these features, however, a much higher $\mathrm{Ca}$ abundance would be required that cannot be accommodated by the later spectra. Therefore, it is likely that the model at this epoch overestimates the ionization, resulting in too much Ca III at the expense of Ca II. The composition used to model this spectrum includes equal parts of $\mathrm{O}$ and $\mathrm{C}$ ( $\sim 45$ per cent by mass), 7 per cent $\mathrm{Ne}$, a total of 1.3 per cent intermediate-mass elements (Mg, Si, S, Ar) including $4 \times 10^{-4}$ per cent Ca. Additionally, we use 0.062 per cent Fe-group elements consisting of 0.05 per cent ${ }^{56} \mathrm{Ni}, 0.012$ per cent 'stable' $\mathrm{Fe}$ (i.e. Fe not produced via the ${ }^{56} \mathrm{Ni}$ decay chain) and traces of $\mathrm{Ti}$ and $\mathrm{Cr}$.

Fig. 2(b) shows the spectrum on 2004 March 29, compared to the model spectrum. The epoch, $6 \mathrm{~d}$ after maximum light, corresponds to $t=20 \mathrm{~d}$ after the assumed explosion date. The model requires a luminosity of $L=3.65 \times 10^{42} \mathrm{erg} \mathrm{s}^{-1}$. The pseudo-photosphere is located at $v_{\mathrm{ph}}=9600 \mathrm{~km} \mathrm{~s}^{-1}$ with a temperature of $T_{\mathrm{BB}}=8060 \mathrm{~K}$ for the underlying blackbody. The composition is similar to the previous model, although less stable $\mathrm{Fe}$ is needed to match the $\mathrm{Fe}$ features because more ${ }^{56} \mathrm{Ni}$ has decayed to $\mathrm{Fe}$.

The next epoch we modelled is 2004 April 7, $15 \mathrm{~d}$ after maximum light and $t=29 \mathrm{~d}$ after explosion (Fig. 2c). The luminosity used in this model is $L=2.97 \times 10^{42} \mathrm{erg} \mathrm{s}^{-1}$ at a photospheric velocity of $v_{\mathrm{ph}}=8100 \mathrm{~km} \mathrm{~s}^{-1}$. The resulting temperature of the photosphere is $T_{\mathrm{BB}}=6640 \mathrm{~K}$. The shell near the photosphere contains still $\sim 84$ per cent $\mathrm{C}$ and $\mathrm{O}$ but somewhat more intermediate-mass elements, and 1.1 per cent ${ }^{56} \mathrm{Ni}$. This spectrum appears to be much redder than the earlier ones. In addition to a lower temperature, blocking by a large number of iron group lines suppresses the UV and blue flux in this and later spectra. The fit to the observed spectrum is acceptable, although the double-peaked re-emission features between 5400 and $6700 \AA$ are too weak in the model. At this and the subsequent epochs, the models also fail to reproduce the absorption features around 6800 and $7200 \AA$. Based on the models, it cannot be uniquely asserted if this discrepancy is due to missing elements in the composition or if the ionization balance is incorrectly determined. The assumption of the thermal photosphere absorption additionally overestimates the flux in this wavelength region.

The following spectrum (Fig. 2d) was taken on 2004 April 14, $22 \mathrm{~d}$ after maximum light and $t=36 \mathrm{~d}$ after explosion. The luminosity used here is $L=2.30 \times 10^{42} \mathrm{erg} \mathrm{s}^{-1}$. The photospheric velocity is $v_{\mathrm{ph}}=6600 \mathrm{~km} \mathrm{~s}^{-1}$, which leads to a blackbody temperature of $T_{\mathrm{BB}}=5950 \mathrm{~K}$. The composition used for this fit is still very similar to the previous epoch, with a slightly higher ${ }^{56} \mathrm{Ni}$ mass fraction (1.6 per cent). The O I feature at $7400 \AA$ appears stronger in the model than in the observation. Unfortunately, the observed spectra show an atmospheric absorption feature at $7500 \AA$ that happens to coincide with the $\mathrm{O}_{\mathrm{I}}$ feature. We decided to use the observed spectrum in which this feature has not been removed because it makes the uncertainty in the shape of the $\mathrm{O}$ I absorption more apparent.

The last epoch we modelled with the photospheric method (Fig. 2e) is 2004 April 20, $28 \mathrm{~d}$ after maximum light and $t=43 \mathrm{~d}$ after explosion. The model requires a change in the abundance pattern. $\mathrm{C}$ and $\mathrm{O}$ are reduced to 10 and 35 per cent, respectively, $\mathrm{Si}$ is increased to 15 per cent and we require 18 per cent of ${ }^{56} \mathrm{Ni}$. The inner boundary of this model is located at $v_{\mathrm{ph}}=5000 \mathrm{~km} \mathrm{~s}^{-1}$. The overall shape of the spectrum is well reproduced, although the velocity of some absorptions is underestimated by the model, indicating that the change in composition may actually occur at somewhat higher velocities than assumed here. The uncertainty in the shape of the density structure in this transition region, however, makes it difficult to find a better match. To improve the fit, we used an intermediate shell at $5800 \mathrm{~km} \mathrm{~s}^{-1}$ that allows us to smoothen the transition somewhat, although the lack of an observed spectrum between April 14 and 21 leaves this shell relatively unconstrained.

\section{MODELS FOR THE NEBULAR SPECTRA}

More information about the properties of the deeper layers of the ejecta can be obtained from nebular-epoch spectra. At late phases, when the optical depth of the ejecta has dropped to below 1, the innermost ejecta produce emission lines, whose strengths and profiles can shed light on the core-collapse process.

The nebular spectra of Type Ic SNe are usually dominated by a strong [O I] $\lambda \lambda 6300,6364$ line, and SN 2004aw is no exception. In the case of the GRB/SN 1998bw, the nebular spectra were used to infer the aspherical distribution of different elements in the ejecta and hence the aspherical nature of the explosion. The narrow [O I ] $\lambda \lambda 6300,6364$ line and the contrasting broad [Fe II] emission suggested a polar-type explosion viewed near the axis (Mazzali et al. 2001; Maeda et al. 2002). For SN 2003jd, which was luminous but did not show a GRB, the double-peaked profile of the [O I] line witnesses the disc-like distribution of oxygen in an explosion similar to that of SN 1998bw but viewed closer to the equatorial plane (Mazzali et al. 2005).

In contrast, other SNe Ic show no sign of asphericity in their late-time profiles. Examples include a low-energy SN such as SN 1994I (Sauer et al. 2006) and a narrow-lined SN with higher energy such as SN 2006aj (Mazzali et al. 2007a). 


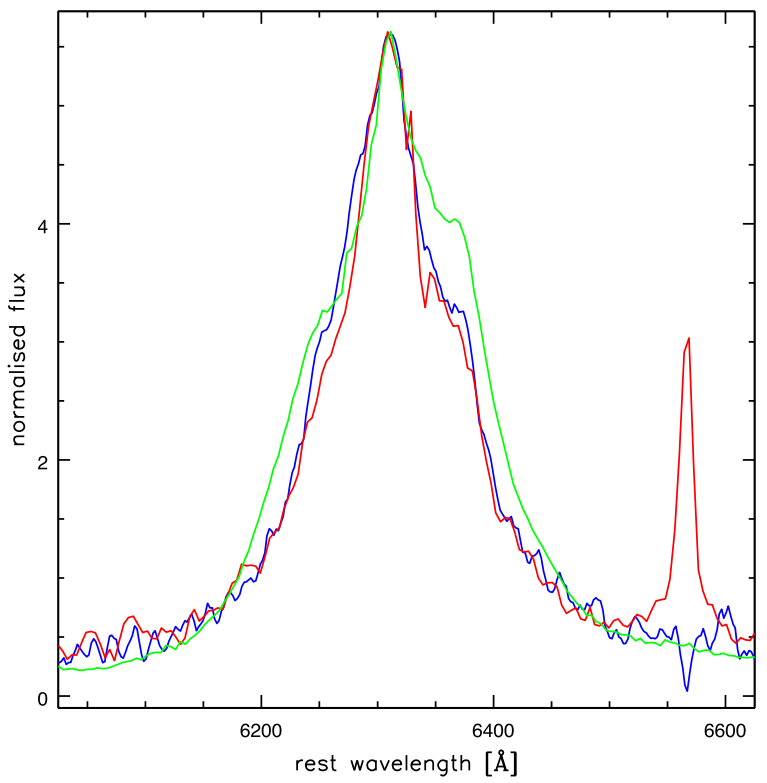

Figure 3. The $\left[\mathrm{O}_{\mathrm{I}}\right] \lambda \lambda 6300,6364$ emission line in the spectrum of $\mathrm{SN}$ 2004aw, $235 \mathrm{~d}$ after explosion (blue line) compared to the same line in the spectrum of the GRB/SN 1998bw obtained on 1999 May 21, 388 rest-frame days after the explosion (red) and that of SN 2002ap obtained on 2002 September 16, $229 \mathrm{~d}$ after explosion (green).

The $\left[\mathrm{O}_{\mathrm{I}}\right] \lambda \lambda 6300,6364$ emission profile in $\mathrm{SN} 20004 \mathrm{aw}$ is remarkably similar to that of SN 1998bw (Fig. 3), indicating that SN 2004aw must have been significantly aspherical. A detailed comparison shows that SN 2004aw has a broader emission base, and the narrow core that characterized SN 1998bw emerges only at low velocities. This suggests a similar morphology for the two SNe, but more extreme in SN 1998bw, and a similar viewing angle. Below we attempt to model the nebular spectrum of SN 2004aw in order to define its properties.

Only the first nebular spectrum of SN 2004aw, obtained on 2004 November $4, \sim 235 \mathrm{~d}$ after maximum, has sufficiently high signalto-noise ratio that several lines can be detected. The spectrum is shown in Fig. 4 along with synthetic models. The emission lines that can be identified are, from blue to red: $\left.\mathrm{Mg}_{\mathrm{I}}\right] \lambda 4571$; [Fe II] multiplets near $5200 \AA$, the strength of which is related to the peak luminosity of the SN; a weak Na ID line at $5890 \AA$; the very strong [O I] $\lambda \lambda 6300,6364$ emission; the strong line near $7250 \AA$, which contains both [Ca II] $\lambda \lambda 7291,7320$ and [Fe II] lines; a weaker Ca II IR triplet near $8600 \AA$, which also contains some $C_{\text {I }}$.

We modelled the spectrum using a code (Mazzali et al. 2001) that computes gamma-ray and positron deposition following the decay of ${ }^{56} \mathrm{Ni}$ to ${ }^{56} \mathrm{Co}$ and hence to ${ }^{56} \mathrm{Fe}$, and then balances the collisional heating induced by the thermalization of the fast particles produced by the deposition with cooling via line emission in nonlocal thermodynamic equilibrium, following the prescriptions of Axelrod (1980). The code can be used in two modes, as discussed in Mazzali et al. (2007a): a one-zone version simply assumes a nebula of constant density, with a sharp outer boundary at a velocity the value of which is selected at input, as are the mass of the nebula and the elemental abundances within it. In a more sophisticated version, the code still assumes spherical symmetry but allows for a radial variation of density and composition, and does not require an outer boundary. In this version, the diffusion of gamma-rays is followed with a Monte Carlo method (Cappellaro et al. 1997). This version is useful to test the prediction of explosion models as well as to

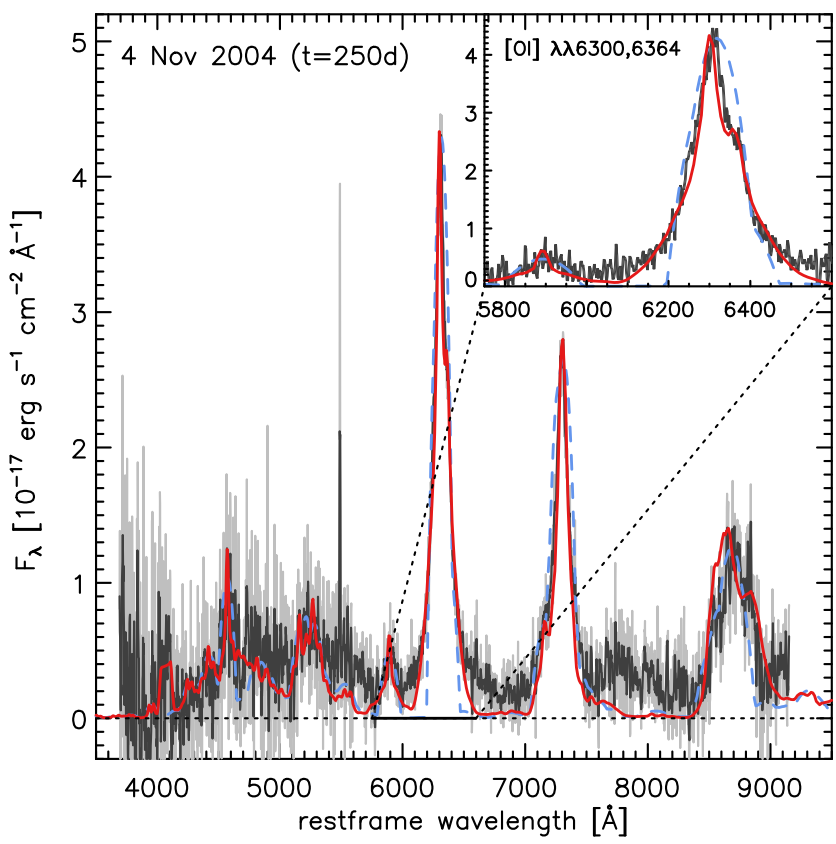

Figure 4. The observed nebular spectrum (light grey line), $235 \mathrm{~d}$ after explosion compared to the model spectra. The one-zone model is indicated by the blue dashed line, and the solid red line represents the improved multizone model. For a better comparison, we also show a smoothed version of the observed spectrum in dark grey. The inset shows a blow-up of the [O I] $\lambda \lambda 6300,6364$ emission.

investigate the radial distribution of mass and abundances in detail when the observed line profiles are sufficiently well determined.

In order to determine the global properties of the nebular spectrum of SN 2004aw, we began by constructing a first, coarse model using the one-zone approach, adjusting the abundances to fit the shape of the prominent emission features. The synthetic spectrum we computed is indicated by a blue dashed line in Fig. 4, where it is compared to the observed spectrum (light grey). The darker line represents the observed spectrum that has been smoothed to emphasize emission features especially in the blue. Assuming a typical rise time of $14 \mathrm{~d}$, we used an epoch of $250 \mathrm{~d}$ after explosion for the calculation. The outer velocity adopted in the calculation that gives a reasonable fit to most emission lines is $5000 \mathrm{~km} \mathrm{~s}^{-1}$. This shows that the nebular spectra originate in a region deeper than the innermost layers studied by means of early-phase spectroscopy, but the separation between the two regions is small. The mass contained within $5000 \mathrm{~km} \mathrm{~s}^{-1}$ in the model is $\sim 1.8 \mathrm{M}_{\odot}$. The dominant element is oxygen $\left(1.3 \mathrm{M}_{\odot}\right)$. The mass of ${ }^{56} \mathrm{Ni}$ required to reproduce the $\mathrm{Fe}_{\text {II }}$ lines and at the same time to excite all other transitions is $0.17 \mathrm{M}_{\odot}$. This is larger than in the low-mass SN Ic 1994I, and similar to energetic, broad-lined SNe Ic such as 1997ef (Mazzali et al. 2000) or 2006aj (Mazzali et al. 2006). The carbon mass is small, only $0.2 \mathrm{M}_{\odot}$, as determined by the strength and shape of the Ca II-dominated feature near $8500 \AA$. A small C/O ratio is quite common in $\mathrm{SNe}$ Ic. The $\mathrm{Mg}$ mass is also small, $0.004 \mathrm{M}_{\odot}$, despite the relative strength of $\left.\mathrm{Mg}_{\mathrm{I}}\right] 4571 \AA$.

The blue dashed line in the blow-up in Fig. 4 shows in detail the [O I] line in the one-zone model. When the observed line profile is viewed in detail, it is clear that it has a composite structure. It shows a broad base, which can be described by a symmetric emission with limiting velocity $5000 \mathrm{~km} \mathrm{~s}^{-1}$, and a narrow core, of width $\sim 2000 \mathrm{~km} \mathrm{~s}^{-1}$, superimposed on it. As we showed above, 
it is similar to the profile observed in SN 1998bw. A similar type of profile was also observed in the BL-SN Ic 2002ap, and it may be interpreted as a signature of asphericity (Mazzali et al. 2007b). As discussed in that paper, the profile can be produced by the superposition of a narrow emission core and either a broad, flattopped profile, which can originate in a shell-like distribution of oxygen, or a double-peaked profile such as what is expected from an equatorial distribution of oxygen viewed on or close to the equatorial plane (Mazzali et al. 2005). The shell+core configuration would be globally spherically symmetric, while the disc+core one would be aspherical. It is not possible to distinguish between these two scenarios based only on the profile of the [O I] line. In the case of $\mathrm{SN}$ 1998bw, the simultaneous observation of broad [Fe II] emission lines favoured the disc+core solution. In the case of SN 2004aw, the Fe lines are not sufficiently well observed for us to be able to determine which scenario is favoured. Still, the similarity of the [O I] profile suggests that the inner parts of SN 2004aw behaved like SN 1998bw. The [OI] emission profile in SN 2002ap was also similar. Interestingly, the [OI] line in SN 2002ap was the broadest of the three SNe. The broad emission base suggests that the intermediatevelocity layers $\left(v \sim 5-10000 \mathrm{~km} \mathrm{~s}^{-1}\right)$ were more spherical in SN 2002ap than in either SN 1998bw or 2004aw. The weaker central emission core also indicates that any central density enhancement was also less extreme.

An aspherical distribution of matter requires a detailed model of the explosion. Here we used our stratified code to test the spherically symmetric scenario. We used the density and abundance distributions obtained from the modelling of the early-time spectra at velocities down to $5000 \mathrm{~km} \mathrm{~s}^{-1}$, the velocity of the photosphere of the last of the early-time spectra (2004 April 20). Therefore, we freely adjusted both the density and the abundances below that velocity, trying to optimize the detailed fit of the line profiles, and in particular that of [O I] 6300, $6364 \AA$ and its narrow core.

The solid red line in Fig. 4 shows the multi-zone synthetic spectrum. The narrow core has a characteristic velocity of $\sim 2000 \mathrm{~km} \mathrm{~s}^{-1}$, and there is a clear discontinuity in line emission at velocities between 2000 and $4000 \mathrm{~km} \mathrm{~s}^{-1}$. In order to reproduce such a feature, it was necessary to introduce a discontinuity in the density profile. The density decreases slightly below $4000 \mathrm{~km} \mathrm{~s}^{-1}$, reaching a minimum at $3000 \mathrm{~km} \mathrm{~s}^{-1}$, and then it increases sharply again in deeper layers. This distribution places a core containing $\approx 0.25 \mathrm{M}_{\odot}$ of material below $3000 \mathrm{~km} \mathrm{~s}^{-1}$. The composition of this core must be dominated by oxygen if the sharp peak of the [O I] line is to be reproduced, but it also must contain ${ }^{56} \mathrm{Ni}$ in order to excite oxygen. The one-zone model already suggests that a large fraction of the ${ }^{56} \mathrm{Ni}$ is located at $v<5000 \mathrm{~km} \mathrm{~s}^{-1}$. As a result, the ${ }^{56} \mathrm{Ni}$ distribution is smooth, peaking at $4000-5000 \mathrm{~km} \mathrm{~s}^{-1}$, but the distribution of oxygen is bimodal: the $\mathrm{O}$ abundance is large outside of $6000 \mathrm{~km} \mathrm{~s}^{-1}$, and again below $3000 \mathrm{~km} \mathrm{~s}^{-1}$. The density structure used to fit the $\mathrm{O}_{\text {I }}$ feature also produces reasonable fits to the other feature, which however are quite noisy. The nebular model includes a total of $0.2 \mathrm{M}_{\odot}$ of ${ }^{56} \mathrm{Ni}$. The total ejected mass is $3.9 \mathrm{M}_{\odot}$, of which $0.85 \mathrm{M}_{\odot}$ are located at $v<5000 \mathrm{~km} \mathrm{~s}^{-1}$.

The density distribution that we finally derived is shown in Fig. 5. It shows the sharp increase of density at the lowest velocities, indicating the presence of an inner region dominated by oxygen.

Classical, 1D explosion models do not predict the presence of any material below a minimum velocity, which represents the position of the 'mass cut': material below this mass cut forms the compact remnant and is not ejected. Light-curve studies had already suggested the presence of an inner dense core of material in some SNe Ic, in particular the massive and energetic ones linked

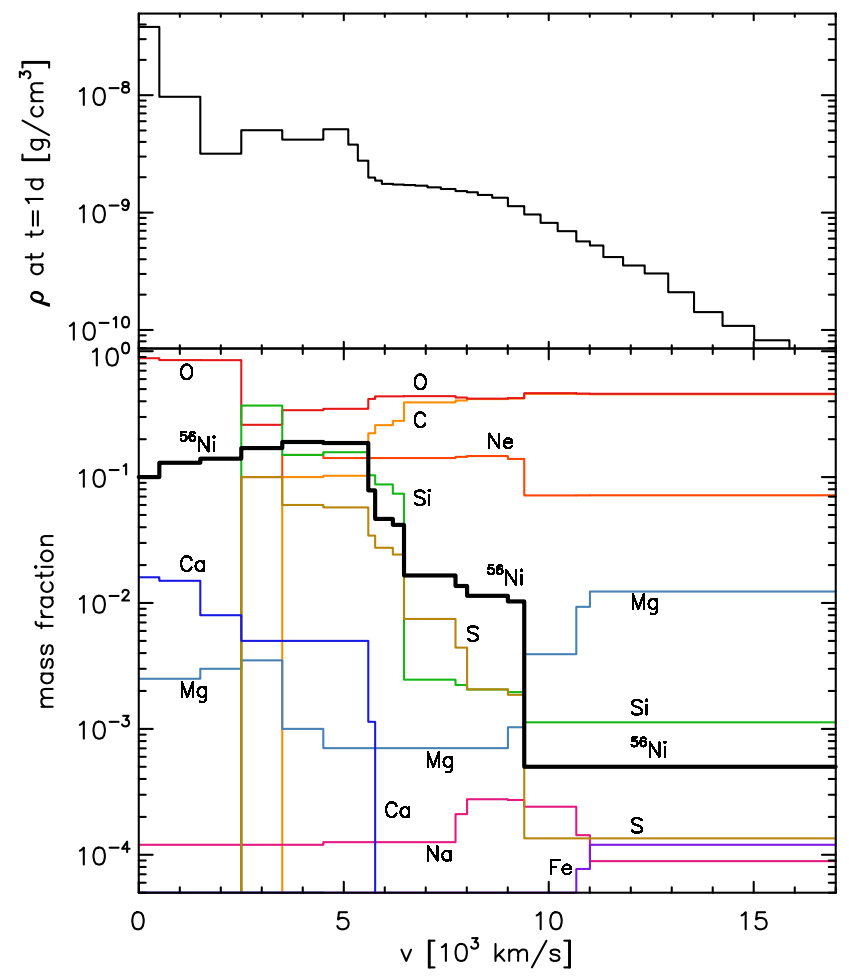

Figure 5. The density (upper panel) and composition structure (lower panel) we used to model SN 2004aw. The nebular spectrum probes the inner part of the ejecta out to $\sim 7000 \mathrm{~km} \mathrm{~s}^{-1}$. The lowest inner boundary for the photospheric spectra is located at $5000 \mathrm{~km} \mathrm{~s}^{-1}$.

to GRBs (Maeda et al. 2003). The nebular spectra of both SNe 2004aw and 1998bw indicate that the inner region is dominated by oxygen, a constituent of the stellar core, rather than by products of nucleosynthesis. The most likely explanation for this distribution of mass and abundances is that the low-velocity material was ejected in the low-energy part of an aspherical explosion. In the case of SN 1998bw, the entire [OI] profile is sharply peaked, indicating a grossly aspherical explosion, while in the case of SN 2004aw, the sharp component of the emission is narrower, suggesting that the asphericity affected only the innermost parts of the ejecta.

\section{BOLOMETRIC LIGHT CURVE}

\subsection{Construction of the bolometric light curve}

We evaluated a pseudo-bolometric light curve of SN2004aw as follows. The magnitudes reported in table 2 of Taubenberger et al. (2006) were dereddened for Galactic extinction using $E(B-V)=0.37$ and the extinction curve of Cardelli, Clayton \& Mathis (1989) and converted to fluxes according to Fukugita, Shimasaku \& Ichikawa (1995). Note that those magnitudes are all corrected for the contribution of the host galaxy, although this was evaluated with different methods for different telescopes: the Katzman Automated Imaging Telescope magnitudes were obtained via aperture photometry on template-subtracted images, while for all the rest point spread function photometry was used within SNOOPY, which includes a correction for the local background.

The light curves in the $J H$ and $K$ bands cover a much more limited time interval than the $U B V R I$ ones; therefore, we assumed that their temporal behaviour at epochs where observations are not available follows that of the $I$ band. Similarly, we assumed that the $U$ - and 


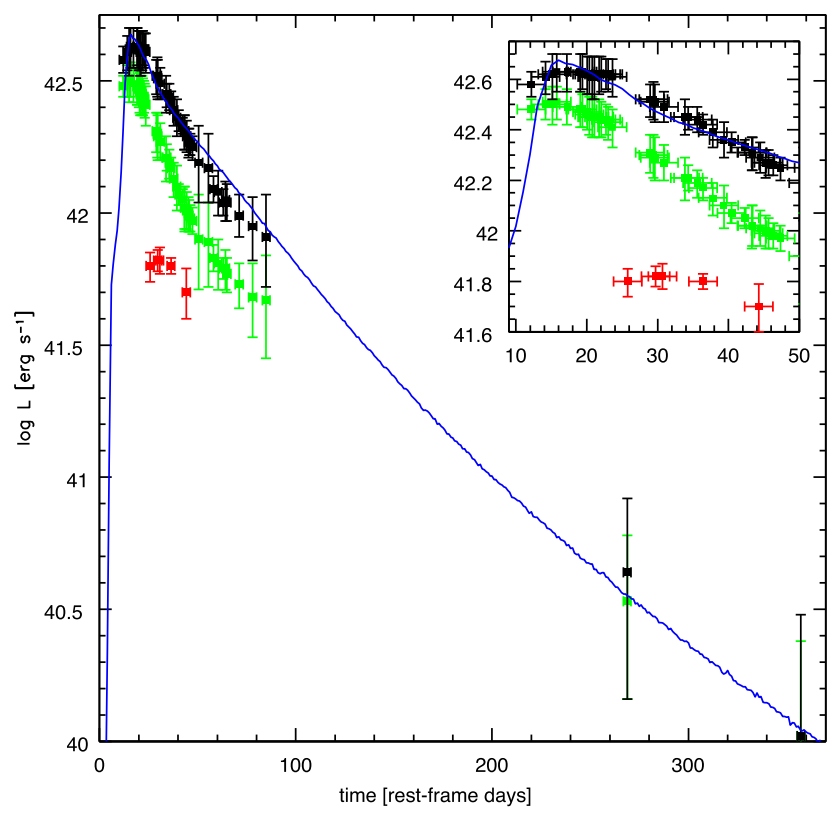

Figure 6. The synthetic light curve computed using the spectroscopic results for SN 2004aw (blue line) compared to the pseudo-bolometric light curve of SN 2004aw (black squares). The optical data used to construct the pseudo-bolometric light curve are shown as green symbols, while the NIR photometry is shown as red symbols. The inset is a blow-up of the peak phase.

$B$-band fluxes at the penultimate and last epochs follow the same general temporal trend as the other optical bands.

The UBVRIJHK monochromatic light curves were splined with a time resolution of $1 \mathrm{~d}$. The monochromatic fluxes in the various bands were linearly interpolated and extrapolated redwards and bluewards of the $K$ - and $U$-band filter boundaries, respectively. Broad-band spectral energy distributions were thus constructed at each epoch and integrated in flux over the range 3000-24 $000 \AA$. Any contribution at wavelengths outside the above range was ignored and is likely not to exceed $5-10$ per cent. The resulting pseudobolometric luminosities corresponding to the epochs of the available photometry are plotted in Fig. 6.

In order to estimate the uncertainties, the errors associated with the optical and near-infrared (NIR) photometry were propagated by adding them in quadrature. For the epochs where only optical photometry was available, the errors on the NIR photometry were estimated based on the errors at the epochs when data were available.

The resulting light curve is consistent with that reported in Taubenberger et al. (2006) when account is taken of the somewhat different treatment of flux at the boundaries of the wavelength range adopted for integration.

\subsection{Light-curve model}

We compare the bolometric light curve obtained as discussed above with models computed with the SN Monte Carlo light-curve code discussed first in Cappellaro et al. (1997) and expanded in Mazzali (2000). Based on a density structure and a composition, the code computes the propagation and deposition of gamma-rays and positrons (using the same description as the nebular spectrum code), transforms the deposited energy into radiation and follows the propagation of the optical photons in the expanding ejecta. Although it is based on simple assumptions about the opacity (Hoeflich, Khokhlov
\& Wheeler 1995; Mazzali 2000), it does yield a reasonable representation of the bolometric light curve (e.g. Mazzali et al. 2013).

A synthetic bolometric light curve was computed for the ejecta density and abundance distribution that was obtained from spectral modelling as outlined above. Above $5000 \mathrm{~km} \mathrm{~s}^{-1}$, the model is a scaled-up version of model $\mathrm{CO} 21$, which gave good fits to $\mathrm{SN}$ 1994I. The photospheric spectra of SN 2004aw resemble those of SN 1994I at similar epochs (with respect to the $B$-band maximum), apart from having higher line velocities. This suggests a similar density structure and a similar mass-to-kinetic energy ratio for both $\mathrm{SNe}$ for the part of ejecta that is responsible for spectral lines in the photospheric phase. Below $5000 \mathrm{~km} \mathrm{~s}^{-1}$, however, we adopted the density derived in Section 3 to reproduce the nebular spectra. The resulting explosion model has an ejected mass of $\sim 4 \mathrm{M}_{\odot}$, an energy $E_{\mathrm{K}} \sim 4 \times 10^{51} \mathrm{erg}$ and a ${ }^{56} \mathrm{Ni}$ mass of $\approx 0.2 \mathrm{M}_{\odot}$.

Fig. 6 shows the synthetic bolometric light curve compared to the observed one constructed as described above. The final bolometric light curve uses as reference the time of $B$ maximum, since the time of the explosion is not accurately known. Bolometric maximum occurs 1-3 d after $B$-band maximum according to our calculation. A pre-discovery limit was obtained on 2004 March 13, which precedes $B$-band maximum by $\sim 10 \mathrm{~d}$. The light curve of SN 2004aw is relatively broad for an SN Ic. The rise time of the $B$-band light curve is $\sim 14 \mathrm{~d}$ for the GRB/SN 1998bw (Galama et al. 1998) and $\sim 9 \mathrm{~d}$ for the XRF/SN 2006aj (Pian et al. 2006), whose explosion dates are known from the accompanying GRBs. In previous theoretical studies of Type Ic SNe, the assumed (or model-constrained) rise time 9-11 d for SN 1994I (Iwamoto et al. 1994; Baron et al. 1999; Sauer et al. 2006) and $\sim 9$ for SN 2002ap (Mazzali et al. 2002), and as much as $20 \mathrm{~d}$ for SN 1997ef (Mazzali 2000). In the case of SN 2004aw, a rise time of 14 observed days is used, which matches the epoch of bolometric maximum in the synthetic light curve.

Although the model does not capture all undulations in the observed light curve, it does reproduce its overall flux level and the time of maximum, indicating that the model that was used has reasonable values of mass, energy and ${ }^{56} \mathrm{Ni}$ mass. We take this as a confirmation of the spectroscopic results. A possible reason for the discrepancies is the approximate treatment of the (grey) opacity, but this was not an issue in other cases (e.g. Mazzali et al. 2013). Another possible source of uncertainty is that we based our calculations on a rescaled rather than a real explosion model, and this rescaling was significant. Thus, our model may not fully capture the properties of a significantly more massive explosion. We therefore use generous error bars on our estimates of mass and velocity.

We can conservatively estimate that, in order to reproduce the bolometric light curve of SN 2004aw, the ejecta mass should be in the range $\sim 3-5 \mathrm{M}_{\odot}$. For smaller masses, we can expect the peak to occur too early, while for larger masses the opposite would be the case.

\section{DISCUSSION AND CONCLUSIONS}

Our models suggest that SN 2004aw was the explosion of a massive $\mathrm{C}+\mathrm{O}$ star that ejected $\sim 4 \mathrm{M}_{\odot}$ of material. If we consider a range of possible remnant masses, from a neutron star with mass $1.5 \mathrm{M}_{\odot}$ up to a black hole with mass $3 \mathrm{M}_{\odot}$, this leads to an exploding CO core of $\sim 4.5-8 \mathrm{M}_{\odot}$. This points to a progenitor star of $M_{\text {ZAMS }} \sim 23-30 \mathrm{M}_{\odot}$, in the context of the pre-SN evolution models of Nomoto \& Hashimoto (1988) and Hashimoto (1995, see also Woosley \& Weaver 1986)). The estimate of $M_{\text {ZAMS }}$ may be modified if the large mass-loss required to remove the $\mathrm{H}$ and $\mathrm{He}$ 


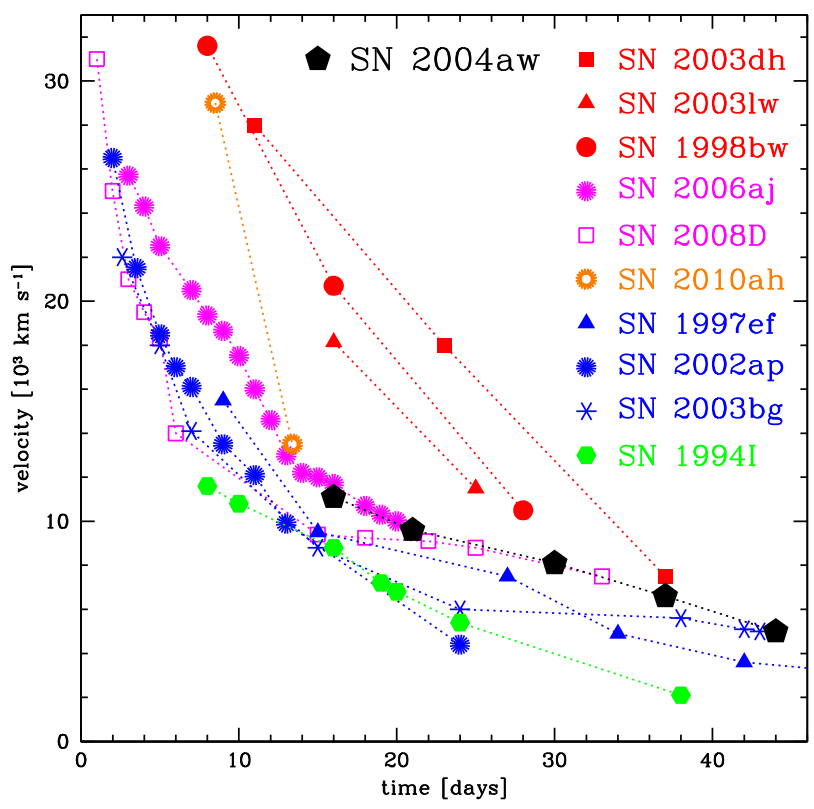

Figure 7. Model photospheric velocities in SN 2004aw (pentagons) and in other $\mathrm{SNe} \mathrm{Ib} / \mathrm{c}$.

envelopes is taken into consideration. For massive single stars, this strongly depends on the uncertain, mass-dependent mass-loss rate in the Wolf-Rayet stage. For example, Woosley, Langer \& Weaver (1993, 1995) found that all their models with $M_{\text {ZAMS }} \gtrsim 35 \mathrm{M}_{\odot}$ evolve to a narrow final $\mathrm{C}+\mathrm{O}$ core mass range of $\sim 2-4 \mathrm{M}_{\odot}$, which may be marginally compatible with SN 2004aw. In contrast, Pols \& Dewi (2002) obtained a final C+O core mass in excess of $10 \mathrm{M}_{\odot}$ for $M_{\text {ZAMS }}>30 \mathrm{M}_{\odot}$ at solar metallicity, adopting updated mass-loss rates, while models with $M_{\text {ZAMS }}<30 \mathrm{M}_{\odot}$ retain a substantial $\mathrm{He}$ envelope before explosion. NIR data of SN 2004aw (Taubenberger et al. 2006) clearly rule out the presence of significant amounts of He (Hachinger et al. 2012). SN 2004aw may have been the outcome of common envelope evolution in a massive close binary, a scenario first proposed by Nomoto et al. (1994, 1995) for Type Ic SNe in general and SN 1994I in particular. This was developed for low-mass $\mathrm{SNe}$ Ic but it may also work at large masses. Alternatively, binary evolution with stable mass transfer may lead to a $\mathrm{C}+\mathrm{O}$ progenitor, but this mechanism may work only at lower masses than what is required for SN 2004aw (Yoon 2015). At high masses, Wolf-Rayet wind mass-loss may strip stars of their outer $\mathrm{H}$ and He envelopes and produce the progenitors of massive SNe Ic.

The explosion of SN 2004aw was moderately energetic, resulting in a kinetic energy $E_{\mathrm{K}} \sim 4 \times 10^{51} \mathrm{erg}$. The ratio of kinetic energy to ejected mass is $\sim 1\left[10^{51} \mathrm{erg}_{\odot}{ }^{-1}\right]$, similar to low-energy explosions such as SN 1994I (Sauer et al. 2006) and significantly smaller than that of low-mass hypernovae such as SN 2002ap (Mazzali et al. 2002). SN 2004aw is often referred to as a broad-lined SN Ic, even though its early-time spectra are quite different from those of hypernovae such as SN 2002ap (Taubenberger et al. 2006) and very similar to those of a low-mass SN Ic like SN 1994I. In Fig. 7, we examine the behaviour of the model photospheric velocity as a function of time compared to other $\mathrm{SNe} \mathrm{Ib} / \mathrm{c}$ with similar modelling. We do not use observed line velocities as those measurements can be highly uncertain because of line blending and broadening, and because line absorption happens at different velocities for different lines. SN 2004aw has no early information but at the times when it was observed the photospheric velocity follows the behaviour of

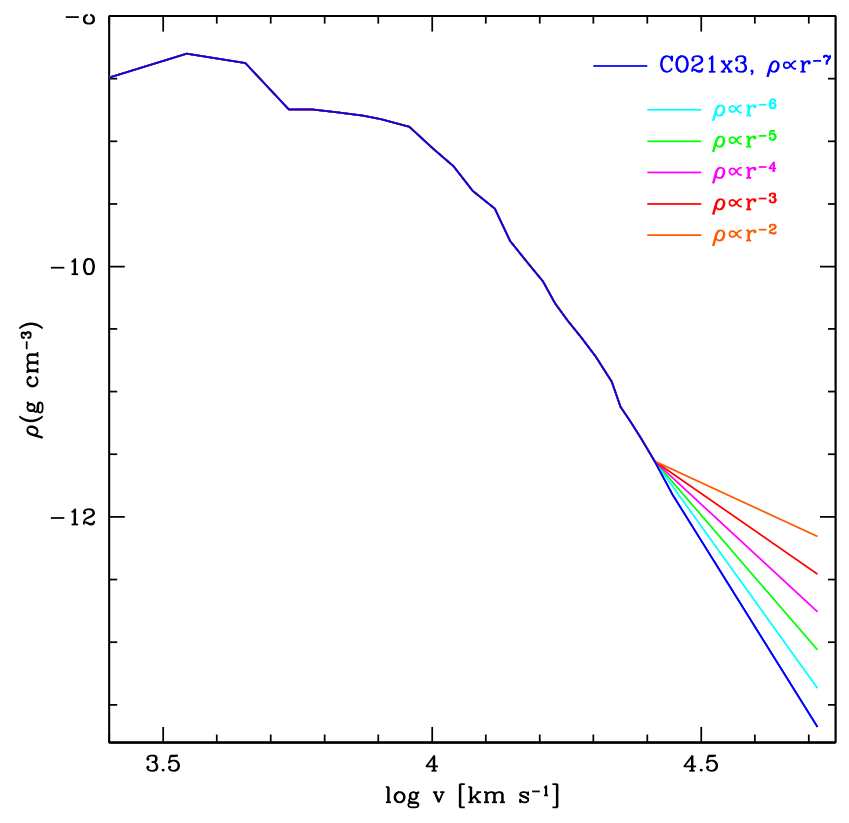

Figure 8. Modified density profiles used to test the earliest properties of SN 2004aw.

SNe like 2006aj or 2008D, which do not have a very large $E_{\mathrm{K}} / M_{\mathrm{ej}}$. SN 2004aw also follows the behaviour of SN 1994I, although it does so at higher velocities.

Could the earlier spectra of SN 2004aw have shown broad lines? We mentioned above that the presence of broad lines is mostly the result of the density slope in the outermost layers. We can modify the density slope in the outermost layers, and test what the spectrum would have looked like at an epoch preceding the observed ones, making sure that the modification does not affect the earliest available spectrum.

Fig. 8 shows the density profiles we have used. We modified the density only above $v=25000 \mathrm{~km} \mathrm{~s}^{-1}$ in order not to affect the models corresponding to epochs where observations exist showing that the lines are not broad. The modified models have different density power-law indices above that velocity, as marked in the figure. Fig. 9 shows the spectra at $7 \mathrm{~d}$ after explosion. Spectra computed with increasingly flatter slopes show broader lines. These lines eventually blend, reducing the number of observed features, as described in Prentice \& Mazzali (2017). Fig. 10 shows the spectra at $15 \mathrm{~d}$ after explosion. At this epoch, all spectra are basically the same, showing that changing the outer density slope at high velocity has no impact at later times.

The models we computed all have $M_{\mathrm{ej}} \approx 4 \mathrm{M}_{\odot}$, but vary in $E_{\mathrm{K}}$ from $4 \times 10^{51} \mathrm{erg}$ for model $\mathrm{CO} 21 \times 3$, which has the steepest outer density slope, to $6.6 \times 10^{51}$ erg for the model with outer density slope power-law index $n=2$. The ratio $E_{\mathrm{K}} / M_{\text {ej }}$ ranges therefore from $\approx 1$ to $\approx 1.6$, which spans some of the range of observed $\mathrm{SN} \mathrm{Ib/c} \mathrm{properties,} \mathrm{although} \mathrm{it} \mathrm{does} \mathrm{not} \mathrm{reach} \mathrm{the} \mathrm{highest} \mathrm{values.}$ If $E_{\mathrm{K}} / M_{\mathrm{ej}}$ was larger, the spectrum at $15 \mathrm{~d}$ would be affected. This sets the uncertainty on the $E_{\mathrm{K}}$ estimate for SN 2004aw, and it is an uncertainty that should be applied to all narrow-lined $\mathrm{SNe} \mathrm{Ib} / \mathrm{c}$ with no early data. It also affects the detailed classification of the SN, as the number of lines, which is 6 at maximum, can be anything between 4 and 6 one week earlier (Prentice \& Mazzali 2017).

Given these uncertainties, we can conservatively estimate that SN 2004aw ejected $4 \pm 1 \mathrm{M}_{\odot}$ of material with a kinetic energy $E_{\mathrm{K}} \approx 4.5 \pm 1.5 \times 10^{51} \mathrm{erg}$. 


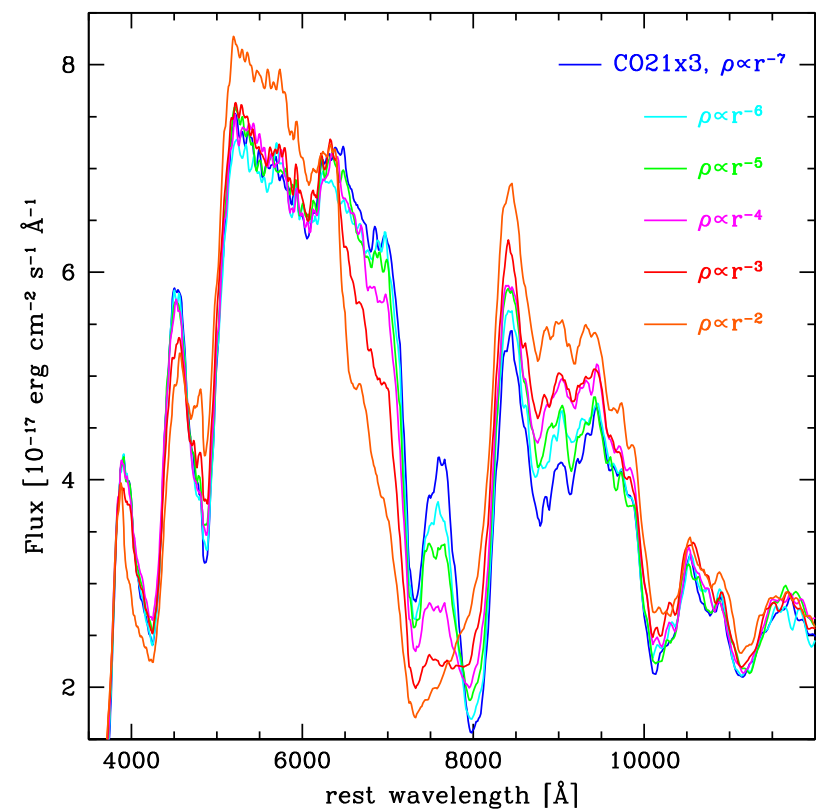

Figure 9. Synthetic day 7 spectra obtained with the different input model density profiles used to test the earliest properties of SN 2004aw.

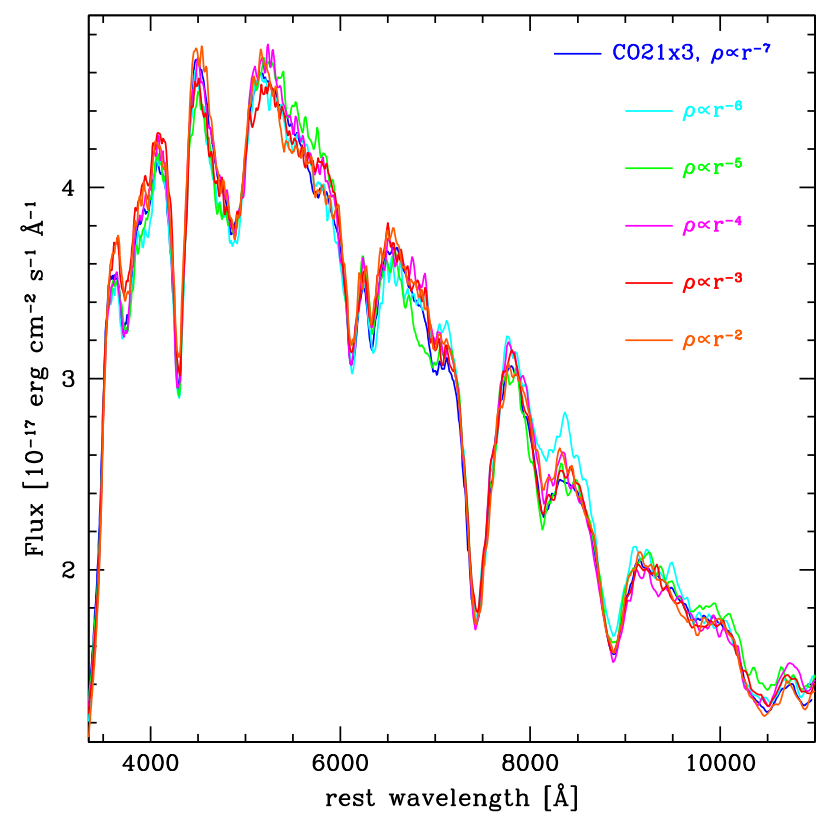

Figure 10. Synthetic day 15 spectra obtained with the different input model density profiles used to test the earliest properties of SN 2004aw.

The value of $E_{\mathrm{K}}$ determined from modelling, although not as extreme as the $\sim 10^{52}$ erg of hypernovae, is probably too large for the canonical 'delayed neutrino-heating' SN mechanism taking place in a proto-neutron star (Janka et al. 2007). In fact, the zeroage main-sequence (ZAMS) mass range of $\sim 23-30 \mathrm{M}_{\odot}$ spans the putative upper boundary for neutron star formation at the end of core collapse and a black hole may be preferred (Fryer 1999). However, doubts have been cast on the sharpness of this separation (Ugliano et al. 2012). If the progenitor core did collapse to a black hole, the SN explosion could have been set off by a central engine comprising of the black hole and an accretion disc as proposed in the collapsar model, providing that the progenitor star was rotating

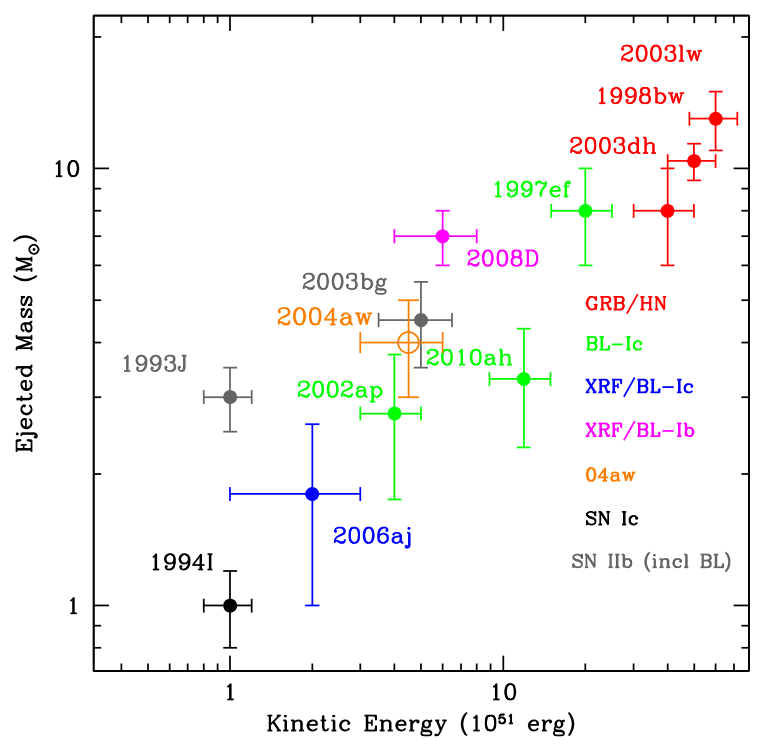

Figure 11. Kinetic energy versus ejected mass in SN 2004aw (circles) and in other SNe Ic.

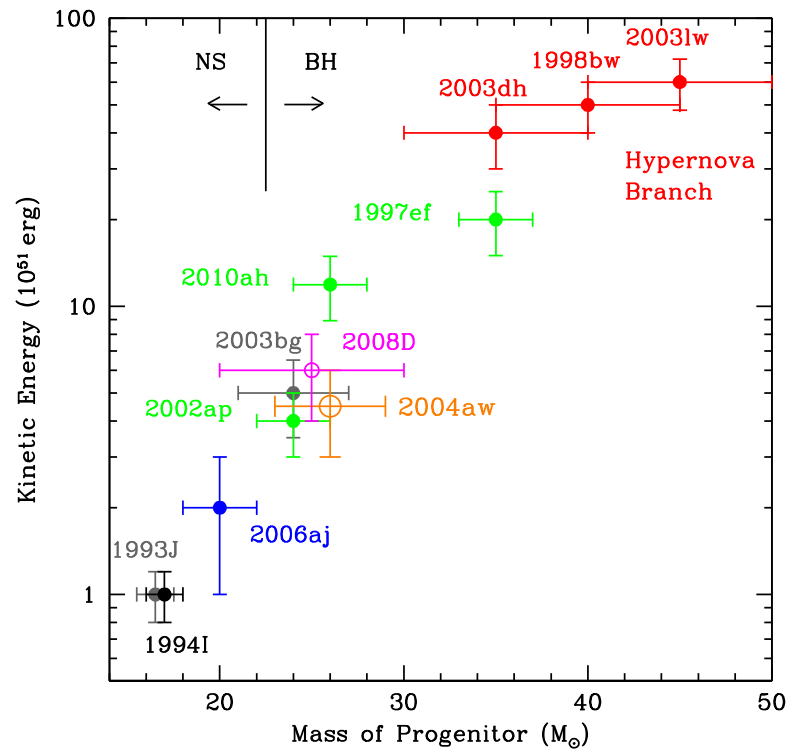

Figure 12. Kinetic energy versus inferred progenitor mass in SN 2004aw (circles) and in other SNe Ic. Colour coding as in Fig. 11.

rapidly (MacFadyen \& Woosley 1999). On the other hand, if the result of the collapse was a neutron star, the explosion could have been aided by a magnetar, which may have injected energy into the SN ejecta, contributed to the synthesis of ${ }^{56} \mathrm{Ni}$, or both (Mazzali et al. 2006a, 2014). Fryer \& Young (2007) simulated the core collapse of a $23 \mathrm{M}_{\odot}$ star and found a long delay to explosion, which may allow time for large magnetic fields to develop in the proto-neutron star.

Adding SN 2004aw to the plots showing the main properties of $\mathrm{SNe} \mathrm{Ib} / \mathrm{c}$ that have been modelled in detail (Fig. 11), we see that it confirms the trend for increasing $E_{\mathrm{K}}$ with increasing ejected mass. This plot is affected for some SNe by the presence of an outer $\mathrm{He}$ shell ( $\mathrm{SNe} \mathrm{Ib})$ as well as some $\mathrm{H}$ (SNe IIb). We can however use the mass of the $\mathrm{CO}$ core to reconstruct the ZAMS mass of the progenitor. Such a plot (Fig. 12) shows a more linear relation between $E_{\mathrm{K}}$ and progenitor mass, although there seems to be some 


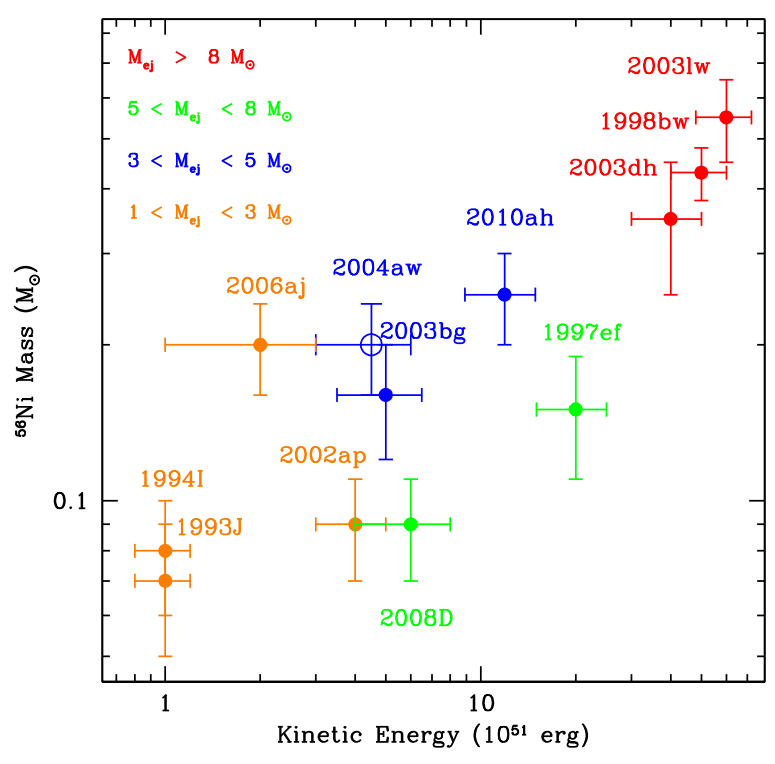

Figure 13. ${ }^{56} \mathrm{Ni}$ mass versus kinetic energy in SN 2004aw (circles) and in other SNe Ic.

spread in the value of $E_{\mathrm{K}}$ at progenitor masses between $\sim 20$ and $30 \mathrm{M}_{\odot}$. Among $\mathrm{SNe}$ in this mass range that have been studied, SN 2004aw has one of the smallest ratios of $E_{\mathrm{K}}$ per inferred progenitor mass, and it is in fact the only one that does not show broad lines in its spectra.

An amount of $\sim 0.20 \mathrm{M}_{\odot}$ of ${ }^{56} \mathrm{Ni}$ was ejected to power the light curve of SN 2004aw. Allowing for the uncertainties in the adopted distance modulus and total reddening (Taubenberger et al. 2006), this value may vary from $\sim 0.15$ to $0.25 \mathrm{M}_{\odot}$. This value lies between the $\sim 0.3-0.6 \mathrm{M}_{\odot}{ }^{56} \mathrm{Ni}$ of the three hypernovae connected with classical long GRBs (SNe 1998bw, 2003dh and 2003lw; Nakamura et al. 2001; Deng et al. 2005; Mazzali et al. 2006b) and the $0.07-$ $0.1 \mathrm{M}_{\odot}$ of the low-energy SN Ic 1994I (Iwamoto et al. 1994) but also of SN 2002ap (Mazzali et al. 2002; Tomita et al. 2006). On the other hand, it is comparable to the value inferred for the Type Ic SN 2006aj, which was accompanied by X-ray flash 060218 and for the non-GRB hypernova SN 1997ef. SN 2006aj has been modelled to have had $E_{\mathrm{K}} \approx 2 \times 10^{51}$ erg and was suggested to have been a magnetar-induced explosion of a star of $M_{\text {ZAMS }} \sim 20 \mathrm{M}_{\odot}$ (Mazzali et al. 2006a), while SN 1997ef had $M_{\text {ZAMS }} \sim 35 \mathrm{M}_{\odot}$ and $E_{\mathrm{K}} \approx 10^{52} \mathrm{erg}$ (Iwamoto et al. 2000; Mazzali et al. 2004). The production of explosively synthesized ${ }^{56} \mathrm{Ni}$ appears broadly to increase with explosion energy (Fig. 13) as well as with ejected mass (Fig. 14), and the relation gets tighter if inferred progenitor mass is used, which eliminates the influence of the outer stellar envelopes on the mass estimate (Fig. 15). Still, in the range of values where SN 2004aw is located, there is significant dispersion. Several factors may be at play: in SN 2006aj, magnetar energy may have contributed to increasing the ${ }^{56} \mathrm{Ni}$ production. In the case of a massive progenitor such as that of SN 1997ef, fallback on to a black hole may have limited the amount of ${ }^{56} \mathrm{Ni}$ that finally was ejected. Other parameters, such as binarity, rotation, metallicity and asymmetry, may affect the outcome of the explosion. This highlights how little we still know about SN Ib/c explosions.

The properties of stripped-envelope core-collapse SNe seem particularly diverse in the progenitor mass range $M_{\text {ZAMS }} \sim 20-30 \mathrm{M}_{\odot}$. While the XRF-SN 2006aj is strikingly similar to SN 2002ap in light curves and spectra, SN 2002ap has no GRB association at all despite being one of the best observed SNe Ic.

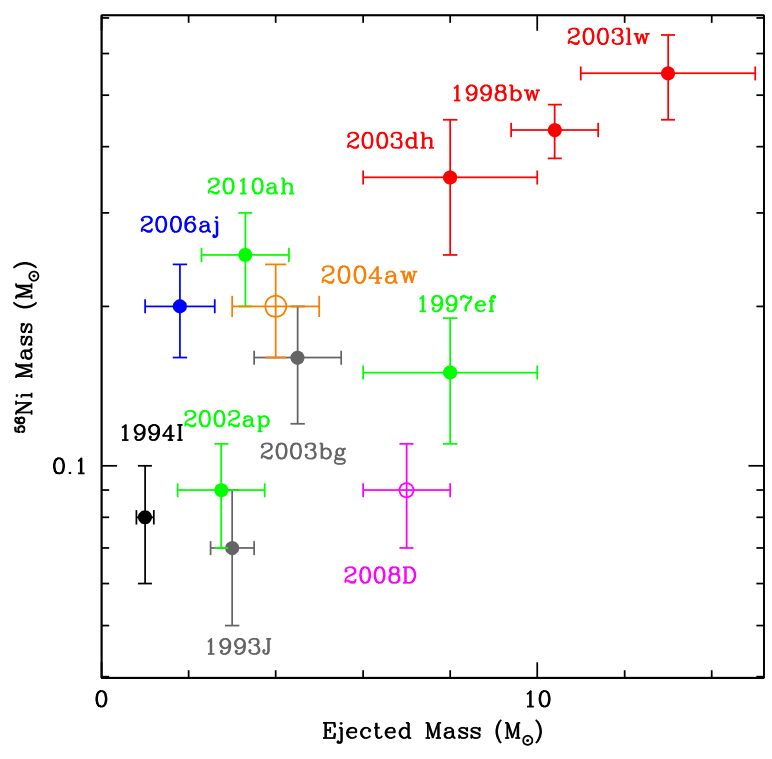

Figure 14. ${ }^{56} \mathrm{Ni}$ mass versus ejected mass in SN 2004aw (circles) and in other SNe Ic. Colour coding as in Fig. 11.

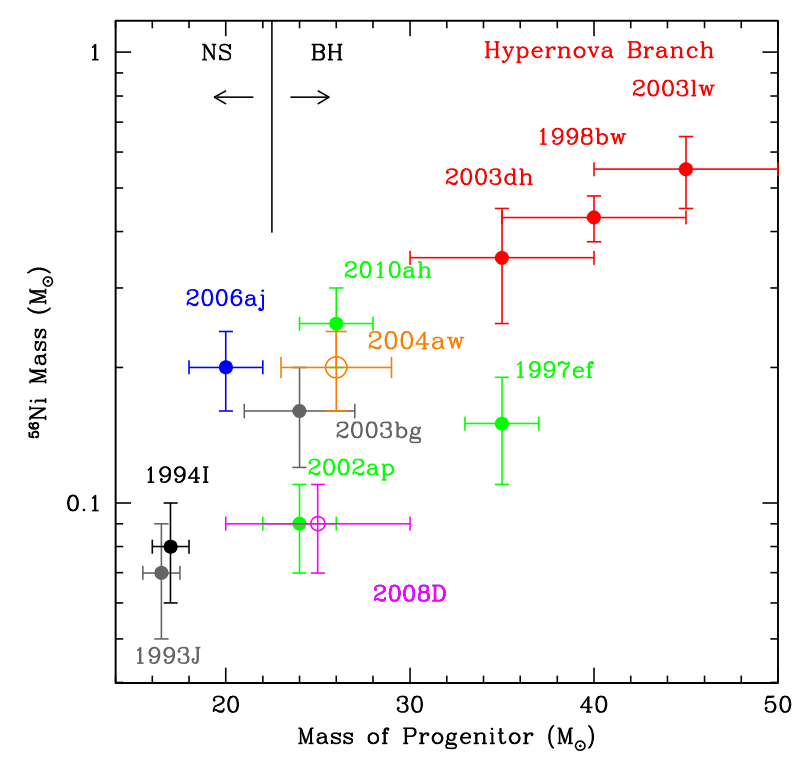

Figure 15. ${ }^{56} \mathrm{Ni}$ mass versus inferred progenitor mass in SN 2004aw (circles) and in other SNe Ic. Colour coding as in Fig. 11.

The Type Ib SN 2008D was captured by the Swift X-Ray Telescope at its very initial flash of soft X-rays (Soderberg et al. 2008) and was soon confirmed in the optical (Deng \& Zhu 2008). With the nature of its initial flash under debate, spectrum and light curve modelling suggest that it had a progenitor with $M_{\text {ZAMS }} \sim 20$ $30 \mathrm{M}_{\odot}$ (Mazzali et al. 2008; Tanaka et al. 2009). Another peculiar SN Ib was 2005 bf ( $M_{\text {ZAMS }} \sim 25-30 \mathrm{M}_{\odot}$; Tominaga et al. 2005) whose composite light curve reached a first peak compatible with moderate ${ }^{56} \mathrm{Ni}$ production and then rose for as long as $\sim 40 \mathrm{~d}$ to reach a second peak, which would require $\sim 0.3 \mathrm{M}_{\odot}{ }^{56} \mathrm{Ni}$. Latetime observations showed that the light curve then traced back the predicted extension of a normal-luminosity SN, suggesting that the second peak was due to the late injection of magnetar energy (Maeda et al. 2007). 
Although our models are 1D, asphericity would probably not change our results much. Asphericity was observed in some Type Ic SNe with no GRB connection, first using polarimetric measurements (e.g. Wang et al. 2001; Kawabata et al. 2002), and then also through revealing line profiles in nebular spectra (e.g. Mazzali et al. 2005; Maeda 2008). The asphericity in SN 2004aw is significant, but it appears to be confined to the deepest layers of the SN ejecta, while in SN 1998bw it affected probably the entire SN ejecta. As already mentioned, what is suggested by the nebular spectra of SN 2004aw is an explosion that was fairly spherical in the outer layers, but significantly aspherical in the deepest parts. It may have been the result of a magnetar or a collapsar that did not inject enough energy to modify the entire SN structure, but did leave an imprint in the regions close to the site of collapse. This may be related to the fact that SN 2004aw had a smaller mass than any GRB/SN. Studies of further examples of energetic SNe Ic are required in order to further our understanding of the presence and impact of an engine.

\section{ACKNOWLEDGEMENTS}

We gratefully acknowledge conversations with G. Meynet during the KITP 2017 Programme on Massive stars. ST acknowledges support by TRR33 'The Dark Universe' of the German Research Foundation.

\section{REFERENCES}

Abbott D. C., Lucy L. B., 1985, ApJ, 288, 679

Ashall C. et al., 2017, preprint (arXiv:1702.04339)

Axelrod T., 1980, PhD thesis, Univ. California, Santa Cruz

Baron E., Branch D., Hauschildt P. H., Filippenko A. V., Kirshner R. P., 1999, ApJ, 527, 739

Ben-Ami S. et al., 2012, ApJ, 760, L33

Bianco F. B. et al., 2014, ApJS, 213, 19

Cappellaro E., Mazzali P. A., Benetti S., Danziger I. J., Turatto M., della Valle M., Patat F., 1997, A\&A, 328, 203

Cardelli J. A., Clayton G. C., Mathis J. S., 1989, ApJ, 345, 245

Clocchiatti A., Wheeler J. C., 1997, ApJ, 491, 375

Deng J., Zhu Y., 2008, GCN Circ., 7160

Deng J., Tominaga N., Mazzali P. A., Maeda K., Nomoto K., 2005, ApJ, 624, 898

Eldridge J. J., Fraser M., Smartt S. J., Maund J. R., Crockett R. M., 2013, MNRAS, 436, 774

Filippenko A. V., 1997, ARA\&A, 35, 30

Fryer C. L., 1999, ApJ, 522, 413

Fryer C. L., Young P. A., 2007, ApJ, 659, 1438

Fryer C. L. et al., 2007, PASP, 119, 1211

Fukugita M., Shimasaku K., Ichikawa T., 1995, PASP, 107, 945

Galama T. J. et al., 1998, Nature, 395, 670

Greiner J. et al., 2015, Nature, 523, 189

Hachinger S., Mazzali P. A., Taubenberger S., Hillebrandt W., Nomoto K., Sauer D. N., 2012, MNRAS, 422, 70

Hashimoto M., 1995, Prog. Theor. Phys., 94, 663

Hoeflich P., Khokhlov A. M., Wheeler J. C., 1995, ApJ, 444, 831

Iwamoto K., Nomoto K., Hoflich P., Yamaoka H., Kumagai S., Shigeyama T., 1994, ApJ, 437, L115

Iwamoto K. et al., 1998, Nature, 395, 672

Iwamoto K. et al., 2000, ApJ, 534, 660

Janka H.-Th., Langanke K., Marek A., Martínez-Pinedo G., Müller B., 2007, Phys. Rep., 442, 38

Kawabata K. S. et al., 2002, ApJ, 580, L39
Lucy L. B., 1999, A\&A, 345, 211

Lyman J. D., Bersier D., James P. A., Mazzali P. A., Eldridge J. J., Fraser M., Pian E., 2016, MNRAS, 457, 328

MacFadyen A. I., Woosley S. E., 1999, ApJ, 524, 262

Maeda K., Nakamura T., Nomoto K., Mazzali P. A., Patat F., Hachisu I., 2002, ApJ, 565, 405

Maeda K., Mazzali P. A., Deng J., Nomoto K., Yoshii Y., Tomita H., Kobayashi Y., 2003, ApJ, 593, 931

Maeda K. et al., 2007, ApJ, 666, 1069

Maeda K. et al., 2008, Science, 319, 1220

Matheson T., Filippenko A. V., Li W., Leonard D. C., Shields J. C., 2001, AJ, 121, 1648

Mazzali P. A., 2000, A\&A, 363, 705

Mazzali P. A., Lucy L. B., 1993, A\&A, 279, 447

Mazzali P. A., Iwamoto K., Nomoto K., 2000, ApJ, 545, 407

Mazzali P. A., Nomoto K., Patat F., Maeda K., 2001, ApJ, 559, 1047

Mazzali P. A. et al., 2002, ApJ, 572, L61

Mazzali P. A., Deng J., Maeda K., Nomoto K., Filippenko K., Matheson T., 2004, ApJ, 614, 858

Mazzali P. A. et al., 2005, Science, 308, 1284

Mazzali P. A. et al., 2006a, ApJ, 645, 1323

Mazzali P. A. et al., 2006b, Nature, 442, 1018

Mazzali P. A. et al., 2007a, ApJ, 661, 892

Mazzali P. A. et al., 2007b, ApJ, 670, 592

Mazzali P. A. et al., 2008, Science, 321, 1185

Mazzali P. A., Maurer I., Valenti S., Kotak R., Hunter D., 2010, MNRAS, 408,87

Mazzali P. A., Walker E. S., Pian E., Tanaka M., Corsi A., Hattori T., Gal-Yam A., 2013, MNRAS, 432, 2463

Mazzali P. A. et al., 2014, MNRAS, 439, 1959

Modjaz M., Liu Y. Q., Bianco F. B., Graur O., 2016, ApJ, 832, 108

Nakamura T., Mazzali P. A., Nomoto K., Iwamoto K., 2001, ApJ, 550,991

Nomoto K., Hashimoto M., 1988, Phys. Rep., 163, 13

Nomoto K., Yamaoka H., Pols O. R., van den Heuvel E. P. J., Iwamoto K., Kumagai S., Shigeyama T., 1994, Nature, 371, 227

Nomoto K., Iwamoto K., Suzuki T., 1995, Phys. Rep., 256, 173

Pian E. et al., 2006, Nature, 442, 1011

Pols O. R., Dewi D. M., 2002, PASA, 19, 233

Prentice S. J., Mazzali P. A., 2017, MNRAS, in press (arXiv:1704:00635)

Prentice S. J. et al., 2016, MNRAS, 458, 2973

Richmond M. W. et al., 1996, ApJ, 111, 327

Sauer D. N., Mazzali P. A., Deng J., Valenti S., Nomoto K., Filippenko A. V., 2006, MNRAS, 369, 1939

Soderberg A. M. et al., 2008, Nature, 453, 469

Stehle M., Mazzali P. A., Benetti S., Hillebrandt W., 2005, MNRAS, 360 1231

Taddia F. et al., 2015, A\&A, 574, A60

Tanaka M. et al., 2009, ApJ, 692, 1131

Taubenberger S. et al., 2006, MNRAS, 371, 1459

Tominaga N. et al., 2005, ApJ, 633, L97

Tomita H. et al., 2006, ApJ, 644, 400

Ugliano M., Janka H.-T., Marek A., Arcones A., 2012, ApJ, 757, 69

Valenti S. et al., 2008, MNRAS, 383, 1485

Wang L., Howell D. A., Höflich P., Wheeler J. C., 2001, ApJ, 550, 1030

Woosley S. E., Bloom J. S., 2006, ARA\&A, 44, 507

Woosley S. E., Weaver T. A., 1986, ARA\&A, 24, 205

Woosley S. E., Langer N., Weaver T. A., 1993, ApJ, 411, 823

Woosley S. E., Langer N., Weaver T. A., 1995, ApJ, 448, 315

Yoon S.-C., 2015, PASA, 32, 15

This paper has been typeset from a $\mathrm{T}_{\mathrm{E}} \mathrm{X} / \mathrm{L} \mathrm{T} \mathrm{E} \mathrm{X}$ file prepared by the author. 